East African Medical Journal Vol. 81 No. 1 January 2004

ANALYSIS OF RUPTURED UTERUS IN DEBRE MARKOS HOSPITAL, ETHIOPIA.

A. Admassu, MD, Doctor of Medicine, Certification in General Surgery, Bethzata Medical Centre, P.O. Box 55, Addis Ababa, Ethiopia

\title{
ANALYSIS OF RUPTURED \\ UTERUS IN DEBRE MARKOS HOSPITAL, ETHIOPIA
}

\section{A. ADMASSU}

Objective: To determine the incidence of uterine rupture in a rural hospital and to examine its cause, type and outcome.

Design: A prospective study.

Setting: Debre Markos Hospital, Ethiopia.

Subject. All patients who were admitted and operated on for uterine rupture in the above mentioned hospital over a period of two years.

Outcome measures: Parity, weather an attempt was made to deliver them preoperatively, diagnosis at admission, vital sign status on admission and type of operation.

Results: There were a total of 1830 deliveries and seventy uterine rupture cases. Frequency of occurrence of uterine rupture was 3.8\% or one in 26 deliveries. The peak incidence was between 25 to 29 years. Mean parity was 3.6 and grand multiparas (>5) accounted for most $(34.2 \%)$. Intraoperatively 62 were found to have complete and eight incomplete rupture with $\mathbf{5 4 . 3 \%}$ anterior rupture. Hysterectomy was done for $\mathbf{5 7}$ and repair for 13 . The postoperative complication rate was $24.3 \%$, sepsis being the most common cause of death.

Conclusion: Uterine rupture is relatively common mainly as a result of obstructed labour and multiparity. Improved healthcare delivery, accesss to prenatal care and family planning will improve the situation.

\section{INTRODUCTION}

Maternal mortality is one of the indices of evaluating healthcare delivery. And it is noteworthy that uterine rupture claims a significant portion of these mortalities in the developing world(1,2). Although inadequancies in the healthcare system are often mentioned as primary reasons, reluctance to seek medical advice or even ignoring and misuse of the existing healthcare infrastructure which mainly stem from illeteracy are contributing factor that should not be underestimated.

Rupture of gravid uterus usually occurs spontaneously caused by cephalopelvic disproportion, malposition and malpresentation. Traumatic rupture occurs following intervention instrumental, manipulatory or pharmacologic. Negligible number of ruptures following augmentation or previous caesarean section still point to the fact that obstructed labour and multiparity remain to be the main causes. This is a reality, which is a direct contrast to causes seen in the developed world (3), where rupture is a rarity.

As to the incidence, uterine rupture is as rare as 1:16849 deliveries in unscarred uterus in USA (3) and as frequent as 1:35 in Jima, and 1:38 in Shashemane (4). The reason for the high frequency in developing countries is that obstructed labour is common compounded by absent prenatal care and unskilled manipulations(1). The objective of this study is to determine the incidence, characteristics of patients, type and cause of rupture, its management and complications. The peculiarity of this study was that cases were handled by general surgeon due to unavailability of obstetrician in the hospital.

\section{MATERIALS AND METHODS}

Debre Markos Hospital is the only hospital in East Gojam zone, which is located in Amhara regional administration about $300 \mathrm{~km}$ north west of Addis Ababa. The hospital receives referred patients from health stations, health centres and an hospital. It is estimated that an average person has to travel a distance of $9.6 \mathrm{~km}$ to reach an health station(5). The hospital has 69 inpatient beds out of which 12 are allocated for Gynaecology and Obstetrics. There is no obstetrician in the hospital, the service being run by a general practitioner and four midwives. There are about 900 deliveries in the hospital each year.

A two year prospective analysis from September 1996 to August 1998 of all laparotomy proven cases of ruptured uterus were done. A protocol was designed for the study which included information like patient age, parity, weather an attempt was made to deliver preoperatively, diagnosis at admission, vital sign status, intraoperative finding, postoperative complication, duration of hospital stay and cause of death. Patients who were referred and who died on arrival were excluded from the study, so were those who were found to have intact uterus at laparotomy. In all patients except one operations were carried out under general anaesthesia. Study was limited to in-hospital stay, none of the patients returned for follow-up.

Complete uterine rupture is defined as tear of the uterine wall with intraperitoneal communication and incomplete 
rupture as tear with intact peritoneum. Parity is defined as delivery of the foetus that reached 28 weeks or more.

\section{RESULT}

Over the study period there were 1830 deliveries and 70 uterine rupture cases operated, making occurences of rupture $3.8 \%$ (one in 26 deliveries). Eight patients died before operation and three were referred with a diagnosis of uterine rupture. The age range was between 18 and 45 years with highest occurrence in the range 25-29 years, $34.3 \%$ (24/70), Table 1. Parity ranged from one to twelve and mean parity was 3.6. Most, $34.2 \%$ (24/70), cases of rupture occured in parafive and above, Table 1. Parity adjusted rate is displayed on Table 2, still those with five or more parity accounted for most of the cases., $12.7 \%$ (24/191)

All the patients who came from outside the town where $31.4 \%$ (22/70) were referred from nearby health stations(5), health centre(9) and an hospital(8). We had $18 \%$ fatality in those referred and $23 \%$ in the selfreferred group. Of the seventy patients only one is reported as having had antenatal care at an health station. In $12.9 \%(9 / 70)$ patients, an attempt was made to deliver before diagnosis of rupture was entertained and non died. One patient is known to have sustained rupture while in hospital following augmentation. The admission diagnosis was different from rupture (e $\mathrm{g}$. intrauterine fetal death, delayed second stage) in $14.3 \%$ (10/70) but only one fatality occurred in the group.

Blood pressure was reported as unrecordable in $35.7 \%(25 / 70)$ patients on arrival to the hospital. These contributed to half, $53.3 \%$, (8/15), of the deaths. Of the total, $58.6 \%(41 / 70)$ received blood transfusion and 12 of them died. Table 3 shows the operative finding with 62 complete and eight incomplete uterine ruptures. The most common was anterior with $54.3 \%(38 / 70)$ lateral 13; posterior ten and anterolateral nine. Only one patient had previous caesarean section that presented with ruptured classical scar. The total number of identified associated injuries were six, five of them being vesico-vaginal communication (four ended up developing fistula) and one isolated extension of the tear into the vagina. The highest number of associated injuries were seen with anterior incomplete tear, three out of six, Table 3. We did not find increased number of associated injuries in those with attempted delivery (two out of six). No foetus was found alive.

Table 1

Cases of ruptured uterus by age and parity. DMH Sep.1996-Aug.1998

\begin{tabular}{|c|c|c|c|c|c|c|c|c|}
\hline \multirow{2}{*}{$\begin{array}{l}\text { Age in } \\
\text { years }\end{array}$} & \multicolumn{6}{|c|}{ Parity } & \multirow[t]{2}{*}{ Total } & \multirow[t]{2}{*}{$\%$} \\
\hline & 0 & 1 & 2 & 3 & 4 & $>5$ & & \\
\hline$<19$ & 2 & 0 & 0 & 0 & 0 & 0 & 2 & 2.9 \\
\hline $20-24$ & 1 & 3 & 0 & 2 & 1 & 4 & 11 & 15.7 \\
\hline $25-29$ & 2 & 6 & 5 & 5 & 3 & 3 & 24 & 34.3 \\
\hline $30-34$ & 1 & 0 & 0 & 4 & 2 & 4 & 11 & 15.7 \\
\hline $35-39$ & 0 & 0 & 4 & 0 & 3 & 7 & 14 & 20 \\
\hline$>40$ & 0 & 0 & 0 & 2 & 0 & 6 & 8 & 11.4 \\
\hline Total & 6 & 9 & 9 & 13 & 9 & 24 & 70 & 100 \\
\hline$\%$ & 8.5 & 12.9 & 12.9 & 18.6 & 12.9 & 34.2 & 100 & \\
\hline
\end{tabular}

Table 2

Parity adjusted rupture rate. DMH Sep. 1996-Aug. 1998

\begin{tabular}{lll}
\hline Parity & No. & $\%$ \\
\hline 0 & $6 / 844$ & 0.7 \\
1 & $9 / 348$ & 2.6 \\
2 & $9 / 219$ & 4.1 \\
3 & $13 / 131$ & 9.9 \\
4 & $9 / 97$ & 9.3 \\
$>5$ & $24 / 191$ & 12.7 \\
\hline Total & $70 / 1830$ & \\
\hline
\end{tabular}


Table 3

Site of uterine rupture and associated injuries. DMH Sep. 1996 - Aug. 1998

\begin{tabular}{llccc}
\hline Site of tear & No. & Complete & Incomplete & $\begin{array}{c}\text { Associated } \\
\text { Injury }\end{array}$ \\
& & Associated injury & No. & 3 \\
Anterior & 33 & 0 & 5 & 0 \\
Posterior & 8 & 0 & 2 & 0 \\
Lateral & 12 & 1 & 0 & 0 \\
Anterolateral & 9 & 2 & 8 & 3 \\
\hline Total & 62 & 3 & & 3 \\
\hline
\end{tabular}

Table 4

Types of Procedure for uterine and their outcome. DMH Sep. 1996- Aug. 1998

\begin{tabular}{lllllll}
\hline Procedure & \multicolumn{2}{c}{ Discharged } & & Died & \multicolumn{2}{c}{ Total } \\
& No. & $\%$ & No. & $\%$ & No. & $\%$ \\
\hline Total Hysterectomy & 45 & 83.3 & 9 & 16.7 & 54 & 77.1 \\
Sub total Hysterectomy & 2 & 66.7 & 1 & 33.3 & 3 & 4.3 \\
Repair & 8 & 61.5 & 5 & 38.5 & 13 & 18.6 \\
\hline Total & 55 & 15 & & 70 & 100 \\
\hline
\end{tabular}

Table 5

Duration of hospital stay for uterine rupture DMH Sep. 1996-Aug 1998

\begin{tabular}{lll}
\hline Days & No. & $\%$ \\
\hline 0.5 & 7 & 10 \\
$6-10$ & 37 & 53 \\
$11-15$ & 19 & 27.1 \\
$16-20$ & 5 & 7.1 \\
$21-25$ & 1 & 1.4 \\
$26-30$ & 1 & 1.4 \\
\hline Total & 70 & 100 \\
\hline
\end{tabular}

Total abdominal hysterectomy was used for most patients, $77.1 \%(54 / 70)$, but repairs $(13$, six with and seven without tubal ligation) and subtotal hysterectomies(3) were also done, Table 4. Associated procedures (e.g. tubal ligation, unilateral salphingooophorectomy) were done for $44.3 \%,(31 / 70)$ and drain (vaginal) was used for $22.8 \%(18 / 70)$.

The operative time ranged between 40 and 150 minutes, average being 77 minutes. In $38.6 \%$ (27/70) it took an hour or less. Interval between diagnosis and operation ranged from two to ten hours, where $55.7 \%$ (39/70) took six hours or less to prepare.

Hospital stay is displayed on Table 5; the average duration was ten days and mode of nine with a range of fewer hours to 28 days. Slightly more than a half, $52.9 \%(37 / 70)$ stayed between six and ten days. Of the total $24.3 \%$ (17/70), acquired non-fatal postoperative complication and wound infection accounted the most, $58.8 \%$ (10/70), while pneumonia, diarrhoea and wound dehiscence occurred in the rest. The case fatality rate was $21.4 \%(15 / 70)$, where seven of them died within four days of admission. Repair contributed the most ratios, $38.5 \%(5 / 13)$. Of the total 55 were discharged improved, Table 4. Out of the discharged ones six left with residual defect (all of them vesico-vaginal fistulae). In two patients the defect was not identified intraoperatively. Sepsis was the most common cause of death with $46.6 \%$ (7/15), one patient died of intraoperative cardiac arrest, while hypovolemic shock and pulmonary edema were the cause for the remaining ones. All the patients were treated with triple antibiotics (chloramphenicol, crystalline penicillin and gentamicin) but there was irregularity in the availability of drugs. 


\section{DISCUSSION}

This study concerns itself with predominantly rural population. As there was no proper obstetric care in the hospital the general surgical team had to accommodate the extra service. It is the background of this inadequate care compounded by common logistic problems that an hospital like ours faces, that these patients were handled. While uterine scar is a leading cause in the western world, obstructed labour and multiparity are uniformly reported from developing countries as causes, which was also a fact in our study $(1,3,4,6)$. Most of the patients seemed to have sustained rupture spontaneously as only $12.9 \%(9 / 70)$ of them have been associated with some sort of intervention in the form of forceps, vacuum, destructive procedure or augmentation. The only post caesarean scar rupture we saw had classical operation which is mentioned more prone to develop this complication than lower segment operation(6). In addition to cases that come to the hospital being complicated, the high number of rupture may be explained by a very small number of deliveries that are conducted in the hospital considering the crude birth rate of the total population. The most common age group where rupture occurred was similar to the age group seen in Shashemane but mean parity was lower and it mostly occurred in grand multiparas(4). This fact is strengthened by the parity adjusted rate where incidence of rupture increases with increase in parity.

It seemed miss diagnosis at admission and intervention that were made before diagnosis did not influence the outcome. Rather severity of condition at admission and type of operation were determinants, supported by the finding that unrecordable blood pressure and repair of a torn uterus were associated with higher number of deaths. Referral from a better health institution did not necessarily mean a better outcome. This may be explained by the fact that patients were relayed from one health instituion to another leading to delay and worsening their conditions. Almost all refered patients arrived at the hospital unaccompanied without any effort to resuscitate them.

Both anterior and lateral tears are mentioned as being common by different authors $(1,6)$. Anterior tear is the most common in our study in contrast to the Shashemane experience. All our patients who developed fistula had anterior lower segment tear. This was as a result of cephalopelvic disproportion which led to impingement of the bladder between the fetal head and pelvis resulting in injury of the bladder that was detected intraoperatively or pressure necrosis that sloughs later on during the postoperative course.

The only treatment option for rupture is surgical. Our decision for the type of procedure was influenced by facts like intraoperative finding, overall condition of the patient and parity. Hysterectomy is the operation of choice in all complicated tears of the uterus(1). Most patients we handled presented late with heavy intraperitoneal contamination dead foetus floating free in the peritoneal cavity, rugged tear edge with hematoma and necrotic tissue. Our effort to preserve the uterus by repairing the tear ended up with disastrous consequences (five deaths out of thirteen) forcing us to take out the uterus almost always in the second year of the study.

Difficulties in organising the operating room, procuring intravenous fluid, blood and other important materials and investigations made it impossible for us to start the operation as early as possible. The above mentioned facts were also noted by Yohannes at Jimma (2), but additionally lack of sence of urgency in the hospital surrounding was a major hindrance.

The rate of postoperative complication especially wound infection can be explained by delayed presentation leading to significant contamination of the operative field, poor postoperative wound handling practice and suboptimal nursing care. The case fatality rate in our study was higher than was found by Bekele(4), and lower than Jima experience(2). In the absence of any form of prenatal care and lack of access to the health care delivery, it will be difficult to make the picture better. Improving the access, provision of health education to increase awareness about prenatal care and family planning, devising an efficient referral system and community based healthcare activities coupled with competent emergency care at higher level health institutions will undoubtedly influence the incidence of this completely avoidable obstetric catastrophe.

\section{ACKNOWLEDGMENTS}

To Dr Yirgu G/Hiwot from the Department of Obstetrics and Gynecology Faculty of medicine AAU, for his valuable advise.

\section{REFERENCES}

1. Heij, H. A. Uterine rupture. Trop. Doct. 1985; 15:127131.

2. Yohannes, Ali. Analysis of maternal deaths in Jima Hospital. Ethiop. Med. J. 1994; 32:125-129.

3. Miller, et al. Intrapartum rupture of the unscarred uterus. Obstetric Gynecology. 1997; 89:671-673.

4. Bekele, C. Rupture of pregnant uterus in Shashemane General Hospital. Ethiop. Med. J. 1995; 33:251-257.

5. East Gojam Department of Planning and Economic development Socio-economic profile of East Gojam. 1992-1994

6. Williams Obstetrics 16th edition. Rupture of the uterus. 861-875. 